Voix et Images

voixetimages

\title{
La Tête de Normande Saint-Onge : le discours "à côté " du narrateur caché
}

\section{Gilles Thérien}

Volume 1, numéro 3, avril 1976

Gérard Bessette

URI : https://id.erudit.org/iderudit/200045ar

DOI : https://doi.org/10.7202/200045ar

Aller au sommaire du numéro

Éditeur(s)

Les Presses de l'Université du Québec

ISSN

0318-9201 (imprimé)

1705-933X (numérique)

Découvrir la revue

Citer cet article

Thérien, G. (1976). La Tête de Normande Saint-Onge : le discours « à côté » du narrateur caché. Voix et Images, 1(3), 448-450. https://doi.org/10.7202/200045ar d'utilisation que vous pouvez consulter en ligne.

https://apropos.erudit.org/fr/usagers/politique-dutilisation/ 


\section{La Tête de Normande Saint-Onge: le discours «à côté» du narrateur caché}

Gilles Carle tient un rôle important dans le cinéma d'ici. Il a acquis le droit, bon an, mal an, de faire des films "commerciaux", c'est-à-dire dont le but avoué est de faire de l'argent, si possible... En même temps, Carle cherche à fabriquer un cinéma qui soit le moins possible guidé par des nécessités commerciales: avoir le droit de faire un cinéma d'auteur pour un public qui va "aux vues». Chacun de ses films se ressent de ce que la balance oscille tantôt d'un côté, tantôt de l'autre. La Tête de Normande Saint-Onge n'échappe pas à ce phénomène et véhicule deux discours, selon les yeux et les oreilles qui sont là.

\section{LA TÊTE}

Le titre du film nous l'indique comme objet privilégié. La fin du film nous la présente en gros plan, sombrant dans un égarement évident. En somme, on pourrait croire que c'était là le but du film. Pourtant le film commençait avec le corps de Normande, corps nu, étalé, montré et vu, et tout au long du film ce corps revient souvent, relié à la danse, aux jouissances sexuelles. On aurait pu, hypothétiquement, appeler le film cle Corps de Normande Saint-Onge". Mais voilà, ça ne se fait pas, le corps semble exclure la tête. Et nous nous installons dans une première dichotomie: tête versus corps. En venant voir la tête, nous aurons «en prime» le corps aussi. Le contraire n'aurait donné lieu qu'à un vulgaire porno dont les têtes sont généralement absentes. Qu'est-ce qu'il y a dans la tête de Normande? Des "bibittes" qui fourmillent, grouillent, chatouillent, empoisonnent sa vie. Son corps connaît aussi le fourmillement, le grouillement et autres "bibittes". Son corps connait le plaisir mais sa tête, non. Ça ne circule pas entre les deux. Le corps/plaisir s'oppose à la tête/folie. Le conflit est ouvert et la tête/folie sera victorieuse. De la dichotomie, nous passons à la séparation. Le corps qui danse n'est pas le corps qui jouit. L'artistesculpteur rapaille les morceaux de ce pauvre Osiris condamné à l'égare- 
ment. Sa tête même n'est pas sa tête, c'est celle de sa mère. II y a osmose entre les têtes comme il y a osmose entre les corps mais pas entre les deux systèmes. Normande semble répéter en son corps le corps de sa mère et en sa tête la folie de sa mère. A la fin du film, Normande n'est plus elle-même. Elle est sa mère. Jamais, en fait, on ne nous a donné le moindre indice que cette belle jeune fille pouvait avoir une folie à elle seule. Elle l'emprunte. Les morceaux épars, montrés en gros plans, la folie, les phantasmes de la fin, autant d'ingrédients qui servent à étab́lir le schéma freudien de cette histoire. Mais il n'y a pas que cela. Les différents personnages masculins créent une dramaturgie qui nous amènera à découvrir un personnage caché.

Au début du film, la relation Normande-Bouliane est établie. Bouliane n'est pas subtil. C'est un étalon. II fait ce qu'il a à faire. II n'a d'ailleurs pas de prénom. II tombe plus facilement alors sous le signe de l'espèce que sous celui de l'individu. II n'est pas utile à la tendresse mais seulement. à. la reproduction. On se demande en quoi il fait «jouir" Normande. Face à lui, elle est celle qui donne tout... mais parce qu'elle le veut bien. Le personnage de l'artiste-sculpteur est aussi fort intéressant. Tous ses désirs passent dans la fabrication de ces poupées à dimension humaine. II a trouvé la pierre philosophale et sait opérer la grande mutation : le sexe devient art (et argent). II a bien un petit sursaut devant une cuisse mais il sait vite revenir à des considérations supérieures. II ne garde pas un vieux fond égrillard qui aurait pu faire de son métier, une occasion... il est castré et sa sublimation est plus un calque de son désir qu'une véritable transformation. Ce n'est pas lui qui va offrir au corps de Normande un petit instant de repos. II parle à sa tête (il fait d'ailleurs dans les têtes, Pie XII, Duplessis...). II est raisonnable, ce qui est le contraire de la folie. II reste un personnage masculin, Carol, magicien, fou et riche. Notons qu'il s'appelle Carol sans "e" ce qui est à peine le masculin de Carole Laure. Carol est une "tapette", dixit Bouliane. S'il ne l'est pas, il n'est toutefois pas entreprenant. II a "séduit» Normande sur le plan de la sensibilité. Ils se comprennent, mais voilà, le magicien qui prévoit, prédit, réorganise le monde d'un coup de baguette est fou... C'est même, à ce titre, une sorte de compagnon de la mère Saint-Onge. Compte tenu de ce que Carle donne à comprendre, Carol, qui a une tête et un corps est fou aussi, à travers une possible perversion. Perversion du corps, il va sans dire. Ce sont les trois hommes qui entourent de façon générale Normande.

Un premier aspect de ces étranges relations apparaît dans un manque d'homme véritable, c'est-à-dire d'un homme avec un corps et une tête, chaque partie assumant les fonctions qui lui reviennent sans qu'il y ait perversion de ces fonctions. Bouliane n'a qu'un corps, le sculpteur qu'une tête et Carol semble pervertir les rôles et fonctions de chacun. En fait, en négatif, nous retrouvons la solitude de Normande, solitude qui deviendra un véritable isolement. Et pourtant, Normande se donne en spectacle. Elle 
danse dans une atmosphère de cabaret, elle regarde la caméra, elle se donne à voir faisant l'amour, etc. Normande Saint-Onge a droit à un traitement spécial dans le film. Alors que les autres personnages sont regardés par un autre personnage - champs/contre-champs - Normande est souvent vue que pour elle-même. L'espace dramatique créé par le champ de la caméra renvoie subitement au réalisateur (et au spectateur qui se tient derrière lui...). Carle devient le quatrième homme, celui qui pousse la tête et le corps à leurs extrémités, folie et orgasme. En retour, it ne donne rien d'autre que ce qu'il prend. Il est, étrangement, dans la situation du voyeur qui prête, pour un instant, le trou de serrure qui comble ses désirs.

L'assimilation que l'on peut faire entre Carol et Carle est lourde de sens. Tous les deux sont magiciens. Tous les deux savent, prédisent et suivent le destin de Normande. Carol, dont on peut enlever le " 0 ", fait des tours de magie. II permet la réalisation des rêves, le retour de la mère et la brusque irruption du quotidien qu'il prévoit (la véritable folie de la mère, les policiers, etc.). Carle, le réalisateur, fait croire à la réalité d'une illusion, centrée sur Normande Saint-Onge. Dans son cas, le magicien disparaît, du moins en apparence. Normande est sa créature. II la rend triomphante puis vaincue. Il lui donne des amours impossibles. II la charge d'une culpabilité sans fond dont on comprend mal l'origine. Il la conduit à la folie mais juste avant de la laisser sombrer, il la montre en lionne devant la victime Bouliane. Lui n'est pas concerné. Il est le triomphateur.

C'est peut-être dans cette situation du réalisateur qui s'inclut sans s'inclure dans la mise en scène que nous trouvons une explication au malaise que laisse ce film. Les faiblesses de l'intrigue et des personnages nous renvoient continuellement au magicien derrière la caméra qui devient ainsi le seul garant de ce qui se passe sur l'écran. Il devient alors évident que le discours du film est un discours à côté, où les têtes deviennent des corps et vice-versa, où la folie et l'orgasme se retrouvent en parallèle, où les personnages sont pauvres de désir et cette perversion du discours renvoie au narrateur caché, à celui qui souffle ce qu'il faut dire, qui permute têtes et corps et qui remplace par son désir le manque qu'il a créé.

C'est la première fois qu'un réalisateur québécois a voulu se mettre en scène par la fiction. Le succès n'est peut-être pas très grand mais la voie est bonne, on le constatera quand il saura sortir du champ de la caméra. 